Meta

Journal des traducteurs

Translators' Journal

\title{
Traduire, adapter, réexprimer
}

\section{Georges L. Bastin}

Volume 35, numéro 3, septembre 1990

La traduction dans le monde hispanolusophone

URI : https://id.erudit.org/iderudit/001982ar

DOI : https://doi.org/10.7202/001982ar

Aller au sommaire du numéro

Éditeur(s)

Les Presses de l'Université de Montréal

ISSN

0026-0452 (imprimé)

1492-1421 (numérique)

Découvrir la revue

Citer cet article

Bastin, G. L. (1990). Traduire, adapter, réexprimer. Meta, 35(3), 470-475.

https://doi.org/10.7202/001982ar

Ce document est protégé par la loi sur le droit d'auteur. L'utilisation des services d'Érudit (y compris la reproduction) est assujettie à sa politique d'utilisation que vous pouvez consulter en ligne.

https://apropos.erudit.org/fr/usagers/politique-dutilisation/
Cet article est diffusé et préservé par Érudit.

Érudit est un consortium interuniversitaire sans but lucratif composé de l’Université de Montréal, l'Université Laval et l'Université du Québec à Montréal. Il a pour mission la promotion et la valorisation de la recherche. https://www.erudit.org/fr/ 


\title{
TRADUIRE, ADAPTER, RÉEXPRIMER
}

\author{
GeORGES L. BASTIN \\ Universidad Central de Venezuela, Caracas, Venezuela
}

«L'honnête homme du XXe siècle (...) ne peut se désintéresser de ce qui touche directement au phénomène de la communication $1^{1}$ » Or, pour reprendre les mots du Don Quichotte canadien, «... au pays de la communication, seul le sens est roi $^{2} . »$

La traduction et l'interprétation de conférence sont au cœur même du phénomène de la communication et ne peuvent d'ailleurs être étudiées et expliquées que par référence à celle-ci. Rares sont en effet les études qui ne font ressortir le double rôle de récepteur et émetteur du traducteur et la responsabilité de celui-ci à l'égard tant de l'auteur original que du lecteur final. Par ailleurs, les chercheurs de l'ESIT (Paris III), outre qu'ils ont concentré leurs efforts sur l'étude du sens, ont mis le doigt sur une étape clé du processus de traduction située entre celles de réception (compréhension) et d'émission (réexpression), à savoir la déverbalisation ou conceptualisation. Une étape sans laquelle la traduction échappe difficilement au transcodage pur et simple.

La liste des travaux consacrés aux phases de décodage, d'exégèse et d'appréhension du sens, bref au passage d'une réalité matérielle (le texte) à une réalité immatérielle (le sens) est longue. En revanche, peu de chercheurs se sont attardés à l'examen du passage du sens abstrait (préexistant ou non) à l'expression concrète. C'est là l'un des aspects de notre recherche sur les problèmes posés par l'adaptation que nous envisageons de présenter ci-après. Par adaptation, nous entendons une traduction soucieuse de la plus grande adéquation possible aux aspirations du lecteur et, partant, concernée par des écarts de forme particulièrement grands qu'impliquent deux réalités socio-linguistiques différentes. C'est le cas notamment des exemples utilisés par les auteurs d'ouvrages de linguistique ou, comme nous le verrons plus loin, d'un ouvrage pédagogique de traduction.

«Tout écrit est réponse à une sollicitation intérieure (...) ou extérieure $(. . .)^{3}$.» S'il est vrai que ces sollicitations sont multiples et qu'aucun écrit ne suppose jamais une connaissance zéro, la production du texte ne se fait pas moins à partir d'un schéma mental. Pottier donne comme point de dépat à ce schéma le monde référentiel; ensuite, par le biais de la perception intervient une conceptualisation (schéma d'analyse) qui, grâce à la compétence linguistique (schème d'entendement), est mise en signes pour aboutir au texte (énoncé discursif) 4 .

En traduction, les mécanismes de réexpression en langue cible ne peuvent être étudiés en référence à la langue de départ (compte non tenu du vouloir-dire) sous peine de succomber à un comparatisme a posteriori qui n'éclairera en rien le processus mis en place. En dépit de l'incidence incontestable de la forme de l'original, la production du texte cible traduit relève bien du passage d'une réalité immatérielle (profonde), le sens, à une réalité matérielle (superficielle), le texte en langue cible. Le passage de la pensée à l'expression, nous l'avons vu, est loin d'être automatique. Il implique un voulu conscient et pensé mis en ouvre par le biais de certains mécanismes. «Il y a réalisation, par le moyen du texte, d'une intention de communication liée à la production d'un effet sur le lecteur ${ }^{5} . \gg$ Nous revoici au cœur de la communication. Et comment ne pas rappeler cette définition de la traduction de D. Seleskovitch: «Faire passer le sens d'un message en produisant le même effet chez le destinataire ${ }^{6} . »$ 
Il s'agira donc, pour le traducteur-producteur d'écrits, d'être particulièrement attentif aux effets de sens. Par exemple, dans un texte sur la dette extérieure du Tiers Monde, faiblesse des pays débiteurs; dans un texte sur l'environnement, antipathie à l'égard des pollueurs (...) Ces effets de sens détectés dans l'original grâce à l'analyse exégétique doivent «donner le ton» de la traduction et s'y retrouver implicitement ou explicitement, selon les moyens d'expression de la langue cible. Ces effets de sens, une fois reconnus, contribuent à la mise en texte dans la mesure où, entre autres, ils déterminent souvent la distribution des rôles, c'est-à-dire les actants qui constituent «les unités sémantiques de l'armature du récit ${ }^{7}$.» Ils s'instituent donc en facteurs de cohésion du texte et seront vus, par le réviseur ou le professeur, comme les premiers critères de fidélité.

Autre point clé de la génération du texte, sa logique. Qui dit logique dit cohérence mais aussi enchaînements ou charnières que le traducteur (générateur de texte) aura le plus grand soin de choisir. Comment effectuer ce choix en dehors du pur respect des normes linguistiques? Vigner affirme: «(...) l'ordonnancement logique (...) se fonde en grande partie sur la connaissance du monde que le scripteur partage avec son lecteur ${ }^{8} . »$ Les traducteurs de l'espagnol savent combien ils recourent à un réagencement logique des idées lorsqu'ils s'adressent à un lecteur francophone. Nous revoici décidément dans l'axe fondamental de la communication, le savoir partagé étant l'un des facteurs primordiaux de la transmission d'idées.

Un tel phénomène se vérifie plus éloquemment encore en interprétation consécutive. La traduction et l'interprétation, bien que différentes dans leur mise en œuvre, relèvent d'un seul et même processus mental symbolisé par le triangle de Seleskovitch ${ }^{9}$. Toute théorisation dans le cadre de l'une de ces disciplines ne peut donc faire l'économie d'une mise en parallèle avec l'autre. Dès lors, de même que Vigner rend compte de la situation d'écriture par un retour constant à la situation de parole, nous pouvons avantageusement tenter de décire l'opération de réexpression du sens en traduction par référence à celle propre à l'interprétation consécutive. Pourquoi ne pas voir dans les «schèmes d'analyse» de Pottier ou dans la «macro-structure textuelle» de Vigner le pendant de la prise de notes de l'interprète consécutif? Prise de notes qui, compréhensible uniquement pour leur auteur, représente le sens sans être linguistique, n'est déjà plus totalement immatérielle et renferme presque toutes les clés nécessaires au déclenchement du discours intérprétatif. Est-ce à dire que le traducteur, procède à l'instar de l'interprète, à une prise de notes? Le parallèle est loin d'être fantaisiste si l'on se reporte à «l'extraction des notions-clés $10_{\gg}$ de J. Delisle.

Ajoutons que d'autres parallèles pourraient être établis entre traduction et interprétation qui concourraient à l'élucidation des mécanismes intervenant dans l'une ou l'autre.

L'adaptation est fondamentalement un problème de réexpression. Le moment est venu d'en mieux cerner l'objet. Le Robert définit «adapter» comme «modifier» (quelque chose) «pour rendre conforme à» et «adaptation (en rhétorique)» comme «le fait de transformer (une ouvre narrative) en conservant la substance narrative et en élaborant la forme convenable au genre choisi (ex. adaptation d'un roman au cinéma)». Vinay et Darbelnet ${ }^{11}$, qui y voient le plus complexe des procédés de traduction, le décrivent comme l'«utilisation d'une équivalence reconnue entre deux situations (ex. la parabole du figuier)». Pour sa part, Vázquez-Ayora affirme: «C'est par le procédé de l'adaptation que la traduction atteint sa vraie valeur et son vrai dynamisme, qu'elle acquiert une «viabilité culturelle...12》 D'autres auteurs se sont prononcés sur ce point. C'est le cas notamment de De Sola, dans un petit ouvrage rendant compte de son expérience de traducteur de Ionescol3. Autre exemple, celui de la préface de Hockett à la version espagnole de son livre A Course in Modern Linguistics ${ }^{14}$. 
Ces définitions, qu'elles soient issues de la lexicologie, de la stylistique comparée, de la littérature ou de textes pragmatiques, confortent notre première hypothèse sur le problème de la réexpression. Dans tous les cas, le point de départ est bien un ou plusieurs effets de sens prédéterminés (n'appartenant donc pas au traducteur mais devant être précisément perçus et rendus par lui). L'étape suivante est celle où convergent les facteurs précédemment nommés: l'ordonnancement logique, la connaissance du monde et le savoir partagé - peut être devrions-nous encore ajouter les techniques de rédaction ${ }^{15}$. L'adaptation, problème de réexpression, résulte ainsi d'un choix, d'une option opérée par le traducteur confronté à une difficulté de reformulation du sens. Une telle convergence a pour seul et unique point le destinataire, sans lequel l'opération totale manquera son seul et unique but: que s'instaure la communication. Nous touchons là notre seconde hypothèse, à savoir que l'adaptation privilégie la langue du destinataire et, en cela, qu'elle est obligatoire. Elle constitue la seule manière de faire passer le sens en produisant le même effet tout en respectant le vouloir-dire de l'auteur. Partant, nous sommes d'avis que le choix du traducteur n'est pas facultatif. C'est ce que laisse entendre assez clairement Vázquez-Ayora ${ }^{16}$.

À ce point, apparaît naturellement la troisième hypothèse: l'adaptation n'a d'autres fonctions et objectifs que ceux-là mêmes de la traduction. Les deux se confondent. La première se confond avec la seconde voire, la dépasse. Elle n'en conserve pas moins un statut particulier du fait de son originalité et de sa complexité.

Voyons comment tout cela se «traduit» dans la pratique.

La traduction en espagnol de L'Analyse du discours comme méthode de traduction pose plusieurs problèmes particuliers et donc une pluralité de choix, notamment: jeter un pont entre deux réalités socio-linguistiques bien différentes et appliquer à la traduction français-espagnol une méthode et des principes conçus pour la traduction anglaisfrançais. De telles difficultés ne sont pas neuves pour de nombreux traducteurs, qu'ils soient littéraires ou pragmatiques. Mais les surmonter implique un choix, une option délibérée du traducteur, principalement en ce qui concerne le traitement des exemples illustrant les propos de l'auteur ou servant de point de départ à une démonstration ou un raisonnement.

L'examen de traductions d'ouvrages semblables à celui de Delisle (c'est-à-dire manuels ou traités faisant référence à deux ou plusieurs langues) révèle en gros deux options. La première consiste en une traduction pure et simple reproduisant les exemples originaux tels quels, les expliquant ou les «traduisant» que ce soit dans le texte ou dans des notes en bas de page avec, comme souci principal, la plus grande fidélité à la langue de l'original. La seconde est l'adaptation (voir note 14) impliquant de nombreux écarts par rapport à cette même langue originale, au service de la fidélité au vouloir-dire (ex.: Jour J = Dia $D$ ) et d'une parfaite adéquation du texte cible au lecteur.

Nous ne retiendrons ici qu'un seul exemple de la première option: la version espagnole du Cours de linguistique générale ${ }^{17}$. S'il est vrai que cette version a permis à des millions d'hispanophones d'accéder aux idées de Saussure, elle n'en est pas moins synonyme de ce que nous avons appelé une «traduction pure et simple».

D'une manière générale, le traducteur espagnol opte pour la reprise des exemples originaux de Saussure auxquels viennent s'ajouter, dans le texte ou en note, une traduction littérale ou une explicitation en espagnol. Par exemple, Saussure parle de «notre $l$ mouillée» (p. 49), ce qui devient: la antigua 1 palatal francesa II castellana (p. 77). Ou le français mouton (p. 160) qui donne El español cannero o el francés mouton (p. 196). Ou encore los niños franceses dicen viendre por venir, mouru por mort, etc., y los españoles dicen cabo por quepo, morido por muerto, etc.» (p. 271) pour «ils disent viendre pour venir, mouru pour mort, etc.» (p. 231). Il n'y a rien à redire à propos de cette façon de 
présenter les exemples de Saussure - certains pourront néanmoins objecter que seule la référence à l'espagnol aurait suffi. Mais le fait d'ajouter la traduction littérale aux exemples originaux présente un inconvénient: cette «traduction», souvent, n'explique rien et peut même induire en erreur. Par exemple, pour illustrer que l'identité phonique n'implique pas toujours correspondance de signification, Saussure donne les exemples suivants : «pomme/paume, goutte/je goûte, fuir/fouir» (p..151) - en espagnol : manzanalpalma, gota/ yo gusto, huir/abrir un hoyo (p. 186). On peut se demander aussi ce qu'explique l'exemple suivant pour un hispanophone: Es que en la frase francesa ces gants son (sic) bon marché (estos guantes son baratos), bon marché est un adjectivo? (p. 188). Ou encore, le fait de présenter tel quel l'exemple original »si je la prends/si je l'apprends» (pp. 146-147 de la version espagnole).

Ce bref examen remet en question, non le talent du traducteur, mais l'option de départ visant à prendre le moins de distance possible vis-à-vis de la langue de l'original au détriment de l'effet créé voire même de l'adéquation aux aspirations du lecteur. Cette crainte des écarts de forme - car c'est bien d'écarts formels dont qu'il s'agit - par rapport au texte de départ nous semble, malheureusement, encore loin d'avoir disparu. Pourtant, aucun traducteur ne contestera que «la traduction part du sens pour aboutir au sens, et le résultat recherché par l'opération tranduisante, c'est-à-dire une équivalence entre deux énoncés de langues différentes - ne s'apprécie lui-même qu'en fonction d'un seul critère: le sens $18_{\gg}$.

Parmi les principes qui régissent la communication, on trouve l'établissement d'un équilibre entre le vouloir-dire de l'émetteur et les attentes du récepteur quant au contenu et au vecteur d'un message déterminé. C'est bien là aussi le devoir du traducteur de contribuer à cet équilibre. La version espagnole d'un ouvrage comme celui de Delisle aura, à n'en pas douter, un destinataire bien différent de celui imaginé ou visé par l'auteur: les «apprentis traducteurs, enseignants et praticiens de la traduction» francophones deviennent hispanophones. De même, le choix délibéré du français au lieu de l'anglais comme langue de départ pour cette initiation à la traduction entraîne des écarts considérables par rapport à la méthode originale. Ces écarts nombreux, tant socio-linguistiques qu'issus d'un couple de langues de travail différent (français/espagnol), nous les avons assumés, dans notre version, sans la moindre hésitation. «Peut-on prétendre qu'il s'agit encore de traduction ${ }^{19}$ ?» En fait, derrière cette question se pointe à nouveau — jusqu'à quand? - le tristement célèbre traduttore traditore dont le seul intérêt est l'allitération contenue dans la formule. Or, quel est l'auteur qui tiendrait rigueur à son traducteur de donner à son labeur une nouvelle ampleur?

Passons à quelques exemples d'écarts relevés dans notre adaptation ${ }^{20}$. Les «livrets d'étudiants» (p. 40) n'existant pas comme tels dans les universités latino-américaines, nous avons parlé de planes de estudios (p. 47). Plus loin, nous avons ajouté, à la liste des «contributions les plus importantes et les plus originales qui ont marqué la recherche en traduction non littéraire au cours du dernier quart de siècle» (p. 49) établie par Delisle, deux ouvrages en espagnol: ceux de Vázquez-Ayora et de García Yebra (p. 62). Ensuite, Delisle rend compte du caractère analogique du Robert (pp. 78-79); nous avons conservé cette description mais en y ajoutant celle des dictionnaires espagnols, analogiques eux aussi, de María Moliner et Casares (p. 112). Puis, dans les notes 14 et 15 (p. 118), Delisle traite du «danger d'atrophie qui menace le français au Canada». Notre version substitue un commentaire sur la langue scientifique espagnole déformée (p. 175). Tous ces exemples, concernent les écarts socio-linguistiques.

Quant aux écarts issus du choix d'un couple de langues différent, on peut mentionner les exemples suivants. Au lieu de citer textuellement l'exemple de Saussure «mouton/sheep-mutton» repris par Delisle (p. 59), nous avons préféré donner la référence 
originale et adapter l'exemple: «poisson/pez-pescado» (p. 79). Ensuite, dans la note 10 (p. 32), Delisle présente des exemples de textes publicitaires en français exploitant des procédés stylistiques. Pour chacun de ces procédés (jeux de mots, répétition, allitération, proverbes adaptés), nous avons retenu des exemples publicitaires vénézuéliens. Les aspects fondamentaux de la méthode préconisée par Delisle sont illustrés par la traduction française d'un texte américain du Time. Notre version, elle, prend appui sur la traduction espagnole d'un texte choisi dans l'Almanach Cousteau. Et finalement, à l'instar de Delisle qui explique et analyse le processus cognitif de la traduction en pénétrant dans le cerveau d'un traducteur français face au titre d'un article du Time, nous avons suivi le déroulement complexe de ce processus mental dans la traduction espagnole du titre d'un article du Nouvel Observateur réunissant les mêmes caractéristiques que le titre choisi par Delisle.

\section{CONCLUSION}

Traduire est loin d'être un exercice intellectuel gratuit. «La traduction a été et reste au service des hommes. Elle n'existe qu'en fonction d'un service à rendre ${ }^{21} . »$ Et qui dit service dit aussi «client», et donc destinataire. C'est là une réalisation incontournable. II s'agit donc bien, une fois le message appréhendé, de le rendre, de le réexprimer pour quelqu'un. La nature, l'environnement socio-linguistique et l'époque de ce «client» déterminent les écarts plus ou moins grands à faire en vue de rendre le message accessible, dans les limites du sens, bien entendu. La difficulté réside par conséquent dans une réexpression attentive aux effets de sens originaux et à la logique et la réalité propres au récepteur, quelles que que soient les distances à prendre quant à la forme dudit message (sens). C'est l'ensemble et l'ampleur de ces écarts qui donnent à la traduction le caractère d'adaptation.

L'étude de cas liée à la version espagnole de L'Analyse du discours comme méthode de traduction révèle que l'option «adaptation» pour ce type d'ouvrage allait de soi. L'apprenti traducteur, l'enseignant et le praticien hispanophones ne peuvent faire les frais d'une traduction pure et simple. Sans quoi, ils sauront faire grief au traducteur de les avoir relégués au rang de spectateurs d'une méthode pédagogique dont ils sont, en fait, les acteurs.

Mais, en fin de compte, sommes-nous en train d'évoquer un procédé ou un processus différent de la traduction? Il s'en faut de beaucoup. Il faut bien passer par l'adaptation si l'objectif est de «faire passer le sens du message en produisant le même effet». L'adaptation est traduction. Ni moins, mais peut-être un peu plus, en ce sens qu'elle incarne et franchit un écart particulièrement grand entre deux réalités socio-linguistiques données ou choisies. Selon l'ampleur de cet écart, la traduction sera adaptation, mais il est difficile d'imaginer que toute traduction, à un moment ou un autre, n'ait pas à franchir toujours une distance temporelle, spatiale ou socio-linguistique considérable.

Traduire, adapter, c'est réexprimer, ce qui veut dire exprimer la même chose d'une façon différente.

\section{NOTES}

1. CERVONI, J. (1987): L'Énonciation, Paris, PUF, p. 8.

2. DELISLE, J. (1983) : Les Obsédés textuels, Hull, éditions Asticou, p. 27.

3. VIGNER, B. (1982) : Écrire, Paris, CLE International, p. 29.

4. POTTIER, B. (1987): Théorie et analyse en linguistique, Paris, Hachette, pp. 106-115. Voir aussi les trois paliers de la géneration du texte chez Vigner, op. cit., p. 43.

5. VIGNER, G., op. cit., p. 29.

6. Cité par A. Hurtado dans La Notion de fidélité en traduction (thèse de doctorat - ESIT-Paris III, 1986), p. 13.

7. GREIMAS, A. J. (1970): Du sens, Paris, Seuil, p. 253. 
8. VIGNER, B., op. cit., p. 42

9. SELESKOVITCH, D. (1984), Interpréter pour traduire, Paris, Didier Érudition, p. 185.

10. DELISLE, J. (1980) : L'Analyse du discours comme méthode de traduction, Ottawa, Éd. de l'Université d'Ottawa, pp. 146-147.

11. VINAY, J.-P. et DARBELNET, J. (1958) : Stylistique comparée du français et de l'anglais, Paris, Dídier, pp. 52-53

12. VAZQUEZ-AYORA, G. (1977): Introducción a la traductologia, Washington, Georgetown University Press, p. 324 (Notre traduction).

13. DE SOLA, R. (1978): «La traducción ? Utopía o reto ?» Cuadernos literarios de la AEV, 1978, $\mathrm{n}^{\circ}$ 145, p. 28.

14. «Dans ce volume, Jorge Suarez et Emma Gregores ont tenté de produire non pas seulement une traduction mais plutôt une adaptation de mon texte $A$ Course in Modern Linguistics publié en anglais en 1958. Je veux dire par là que, où cela était possible, les exemples basés sur l'anglais et les langues germaniques ont été remplacés par des exemples basés sur l'espagnol et les langues romanes. C'est pourquoi je suis convaincu que le lecteur, conscient de l'énorme difficulté de cette entreprise se joindra à moi pour féliciter Mme Gregores et M. Suarez d'avoir mené à bien cette tâche difficile et précieuse.» (Notre traduction)

15. HOCKETT, Ch. (1971), Curso de linguística moderna, (deuxième édition), Buenos Aires, EUBA.

16. DELISLE, J. : «La traduction de textes pragmatiques est un art de ré-expression basé sur les techniques de rédaction.»

17. VAZQUEZ-AYORA, G. : «Le traducteur doit savoir que la compréhension d'une æuvre ne dépend pas seulement du niveau d'intelligence du lecteur mais bien des principes qui régissent la communication...», pp. 322-323.

18. De SAUSSURE, F. (1978), Cours de linguistique générale, Paris, Payot.

De SAUSSURE, F. (1945), Curso de linguística general, Trad., prólogo y notas de A. ALONSO, 11ed., Buenos Aires.

19. PERGNIER, M. (1983): «Quelques considérations sur l'équivalence sémantique», Revue de phonétique appliquée, nos $66-67-68$, p. 132.

20. CARY, E. Comment faut-il traduire?, p. 66. Les pages renseignées pour les exemples espagnols sont celles de l'exemplaire de la version espagnole déposé à l'Universidad Central de Venezuela (UCV-Caracas), les autres renvoyant à l'ouvrage de Delisle.

21. CARY, E., op. cit., p. 85. 